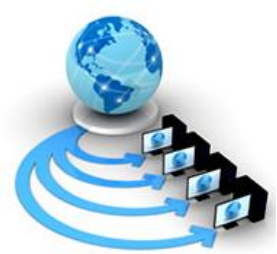

Volume 11, No. 2, March-April 2020

ISSN No. 0976-5697

International Journal of Advanced Research in Computer Science

RESEARCH PAPER

Available Online at www.ijarcs.info

\title{
A NOVEL ROUTING PROTOCOL IN WIRELESS SENSOR NETWORK'S (WSN) BASED ON CLUSTER OPTIMIZATION
}

\author{
Putri Kevin \\ Brahijaya University \\ Department of Computer Science \\ Indonesia
}

\begin{abstract}
Smart Electric Grids are authentically tracked and managed using Wireless Sensor Networks (WSN) for different applications in the field of Smart Grid applications. The purpose of this paper is to simulate and scrutinize the performance of four WSN Routing protocols operating in various smart grid environments. The four WSN routing protocols that will be analyzed in this paper are the Low Energy Adaptive Clustering Hierarchy (LEACH), Centralized LEACH (LEACT-C) and two conventional protocols namely Minimum Transmission Energy (MTE) and Static Clustering. It is important to select and use the appropriate WSN routing protocol for smart grid applications and this study provides an understanding of how each protocol perform under different environments. The results of the study have shown that the clustering approach is more reliable than the conventional protocols namely static clustering and MTE. Rather, uniform dissipation of energy through the sensor nodes was observed in the clustering approach which is important as it increases the lifetime of the networks through less energy consumption.
\end{abstract}

Keywords: wireless sensor network, energy consumptions, clustering, Routing, sensor, location

\section{INTRODUCTION}

Grids are considered as a renovated method of electric power grid technology which uses sensors with advanced features and developed distribution computing technologies. They act as a solution for most of the problems that must face when using electric power grids such as lack of communication, automation, diagnosis of faults and surveillance. When compared with the electric power grids, smart grids only require online tracking, diagnostics, and protection to make sure efficient monitoring using integrated automation and self-recovery options. WSNs have been identified as an optimistic method for energy-efficient, ideal and productive ways of controlling smart grids with low-cost operation in recent times[1-2, 20]. However, several challenges have to be faced when using WSNs because of the unique features they opt to have, resource limitations, and complex electric-power environments. This study will be useful to understand the performance and compatibility of WSNs in different operating environments because WSNs bring enormous benefits for the smart grid technology despite all these challenges.

Sensors are used to collect local information from the physical environments to facilitate the decision-making process [3]. They are self-powered devices yet small and cheaper and have the capability of communicating with remote systems using external networks. There are three ways how WSNs send data using the routing protocols to the base station (BS).

- Direct Communication (DC)[4]: The sensor nodes are capable of sending the data directly to the BS and are known as the simplest protocol. In DC, the nodes which are far away from the BS send data faster than others because they dissipate data faster to the BS.
- Minimum Transmission Energy (MTE) Protocol [5]: The node acts as a router for the other nodes because the nodes send data to the BS through intermediate nodes [6].

- Clustering protocols: The wireless medium, the lower energy supply of the WSN nodes, and the requirement for self-configuration have caused problems regarding energy efficiency and the lifetime of the networks. Rather, the network protocols were unable to keep as less-weighed as well [7]. Therefore, in clustering protocol [8], the nodes are arranged as independent clusters and allow the data to be transferred to the cluster head $(\mathrm{CH})$, then to the BS where the data are collected and processed to allow the end-user to access the information [9].

The Low Energy Adaptive Clustering Hierarchy (LEACH) is a cross-layer protocol architecture [10] which is also known as a cluster-based hierarchical algorithm that incorporates media access which is also an energy-efficient cluster-based routing with application-specific data to accomplish better performance. In LEACH, the nodes form decisions by themselves without any intermediate contribution. This protocol is energy efficient because the $\mathrm{CH}$ dissipates energy uniformly throughout the network.

LEACH-C is slightly different from LEACH [11] as it uses central BS to determine the formation of good clusters, $\mathrm{CH}$ and even distribution of energy in each cluster. A cluster below the average energy level is not allowed as a $\mathrm{CH}$ in the next round. The ability of other nodes to communicate with the BS will be disabled once the energy of the $\mathrm{CH}$ gets eventually exhausted. Simplified propagation models that were used by radio propagation models were used to evaluate the performance of these protocols [12].

The normal shadowing path loss model is a reliable channel model to use in contrasting these routing strategies and it has 
been shown by the power grid environments were designed utilizing WSNs compatible with IEEE 802.15.4.The results showed that the radio transmission conditions of the smart grid had a significant impact on the performance of all protocols. The results of this study have shown that the performance observed in the protocols used in the study have been affected by radio transmission conditions of the smart grid.

\section{WSN Routing Protocols}

WSNs can be used to find the most reliable pathway for data to be sent over a network. The four protocols used for this study are explained below.

\section{A. Minimum Transmission Energy (MTE)}

This is a multi-hop routing protocol [13] where the nodes can directly collaborate with other nodes within the radio transmission range. The special feature in this protocol is that there is no need to compute new routes once a node dies because the nodes surrounding the dead node send their data to the neighbor nodes to secure a seamless flow of data. This occurs by the Carrier Sense Multiple Access protocol (CSMA) MAC protocol and continues until the data are received by the BS.

\section{B. Low energy adaptive clustering hierarchy (LEACH)}

In this protocol, data is allowed to send via the nodes to the $\mathrm{CH}$ where these data are transferred to the BS to be processed. In $\mathrm{LEACH}$, the $\mathrm{CH}$ is chosen randomly to facilitate even distribution of energy in the network. Network lifetime has rounds which are definite, disconnected time intervals and each round has two phases namely setup phase and steady-state phase [14]. In the setup phase, clusters are formed, and the $\mathrm{CH}$ is chosen. A node cannot become a $\mathrm{CH}$ again once chosen as $\mathrm{CH}$ for a given number of rounds. A stochastic algorithm is used to elect a node as $\mathrm{CH}$ or not.

The Low Energy Adaptive Clustering Centralized Hierarchy (LEACH-C) [15] can be adapted to the uneven and vibrant dispersion of energy between the sensor nodes. Once elected, the $\mathrm{CH}$ broadcasts an advertisement message to the whole network and the other nodes determine their $\mathrm{CH}$ according to the signal strength of the advertising message. After determining their $\mathrm{CH}$, the nodes begin to send messages to the $\mathrm{CH}$ to register and afterward they can communicate and send data to the $\mathrm{CH}$. In the steady phase, the $\mathrm{CH}$ aggregates all the data and sends it to the BS. The process of electing a $\mathrm{CH}$ for the next round will continue. The randomization process takes place to rotate the CHs in the LEACH protocol.

\section{Static routing protocols}

Simulated annealing algorithm as in LEACH-C is used to form clusters between nodes that are not within the communication range. Cluster head $(\mathrm{CH})$ is chosen to remain active throughout the lifetime and clusters are chosen a priori. This protocol is very much similar to LEACH-C except the clusters are chosen a-priori. Here. The data communication is scheduled from cluster members to the cluster head. The cluster head is marked with data aggregation. However, the chosen static cluster of this protocol is subjected to death because the cluster head and the cluster remain unchanged during each round. Therefore, the lifetime of the network cannot be escalated. Static routing is also used in many other areas of sensor network like localization where static based system is adapted to enforce the routing strategies [16].

\section{Low energy adaptive clustering hierarchy centralized}

The process of electing a $\mathrm{CH}$ takes place with the following assumptions.

- All nodes of the network have the same amount of energy.

- All nodes have data to be sent to the $\mathrm{CH}$

However, this is not valid for instances when the energy of the nodes differs according to the role. The communication distance between the clusters depends on the position of the cluster head. Also, the energy consumption between the clusters is determined by the distance between the clusters. Accordingly, clusters that have a greater distance between them consume more energy and clusters which consume more energy are more likely to become a $\mathrm{CH}$. Therefore, in $\mathrm{LEACH}$, the $\mathrm{CH}$ is selected according to the energy the clusters possess comparative to the overall energy of the other clusters in the network rather than electing $\mathrm{CHs}$ according to the number of rounds. Therefore, each cluster should send its location to the $\mathrm{BS}$ to determine the $\mathrm{CH}$ considering the average energy of each cluster. This is achieved using activating a global positioning system receiver when each round starts. The BS analyzes these details and sends IDs of the $\mathrm{CHs}$ that have been chosen to the network. If a node identifies its ID in this message, it gets to know that it has to perform as the $\mathrm{CH}$ while other nodes go to sleep until the transmission time [17]. The steady phase of LEACH-C is identical to that of LEACH [18].

\section{Proposed Model AND Simulation Results}

To simulate the entire structure of the routing protocols we have simulated all the WSN routing protocols in favored environment. The NS2 simulator is being used for initial computation in a timed manner. The total time is considered to be $12.5 \mathrm{Nn} \mu \mathrm{s}$ taken from [19] where the $\mathrm{s}$ indicates the no of sequential slots. The overall propagation is considered as 2.5 from [19] for almost 1000nodes deployed in $1000 \mathrm{~m} \times 1000 \mathrm{~m}$. Table 1 shows the common simulation environments along with their numeric values. The data items and length are 1000 bytes/ transferred message with a wider header of 50 bytes.

\begin{tabular}{|l|l|l|}
\hline Simulation Environment & $\begin{array}{l}\text { Path } \\
\text { loss }\end{array}$ & $\begin{array}{l}\text { Routing } \\
\text { deviation }\end{array}$ \\
\hline $\begin{array}{l}\text { 1000-kv smart grid in outdoor } \\
\text { environment }\end{array}$ & 3.42 & 4.42 \\
\hline $\begin{array}{l}\text { 100-kv smart grid in outdoor } \\
\text { (NLOS) }\end{array}$ & 4.51 & 3.95 \\
\hline General pathloss exponent & 2.45 & 3.54 \\
\hline Power loss & 2.64 & 4.23 \\
\hline Time span & $12.5 \mathrm{nms}$ & $11.5 \mathrm{nms}$ \\
\hline
\end{tabular}

Table 1 Simulation parameter

The number of packets received at each node is most important factor with time span and total energy loss. The values are recorded at different time. The main idea that needed to considered is a data packet received at base station to all the sensor nodes. To check the data and network authenticity we need to record the data packet at each node. If all the nodes are getting enough packets it means the network is stable and provide full network coverage. The average energy is again computed on each node. The path loss, power loss on grid and initial level to final level of energy is also 
measured and recorded in the data base. Let us suppose the $e l_{i}$ and $e l_{f}$ are the initial and final energy level at each node of WSN. The total energy consumption is then computed as follows:

$$
E_{f}^{i \rightarrow n}=\frac{\sum_{i}^{k}\left(e l_{i}-e l_{f}\right)}{\sum N}
$$

The active number of nodes is another parameter that is considered while performing the network routing. The total performance of network and nodes is always depended to the activities of the nodes. The lower value of node in terms of energy and highest values indicate the activeness of the nodes in certain factors. As we are simulating all the nodes and routing in NS2, so the parameters of the nodes are also considered according to NS2 structure as shown in Table 2.

\begin{tabular}{|l|l|}
\hline Simulation Environment & Path loss \\
\hline $\begin{array}{l}\text { 1000-kv smart grid in outdoor } \\
\text { environment }\end{array}$ & 3.42 \\
\hline $\begin{array}{l}\text { 100-kv smart grid in outdoor } \\
\text { NLOS) }\end{array}$ & 4.51 \\
\hline General pathloss exponent & 2.45 \\
\hline Power loss & 2.64 \\
\hline Time span & $12.5 \mathrm{nms}$ \\
\hline Energy at the start & $0.5 \mathrm{Jouls}$ \\
\hline Energy at peak stage & $1.2 \mathrm{jouls}$ \\
\hline Protocol Antenna gain & 2 \\
\hline Hight of antenna & $3 \mathrm{~m}$ \\
\hline Time span of simulation & $500 \mathrm{~s}$ \\
\hline Base station spherical location & 60989 \\
\hline Wavelength & $0.987 \mathrm{~m}$ \\
\hline
\end{tabular}

Table 2 NS2 simulation parameter setting

With the presence of ideal propagation all the protocols were simulated and then we have compared whole simulation by using shadowing model. In WSNs ray model is widely used which provide a simple assumption and proper fading modeling, which utilized less energy and provide more data sources. We also investigated that in the SCADA systems of $1000 \mathrm{kv}$ electric system the ray model has high impact and accuracy with all the LEACH protocols. While in presence of shadowing model the accuracy and impact is always very low utilizing high amount of energy. This is due to having a lot of RSSI data sets from the deployed nodes. The performance of LEACH and LEACH-C protocol is shown in Figure 1.

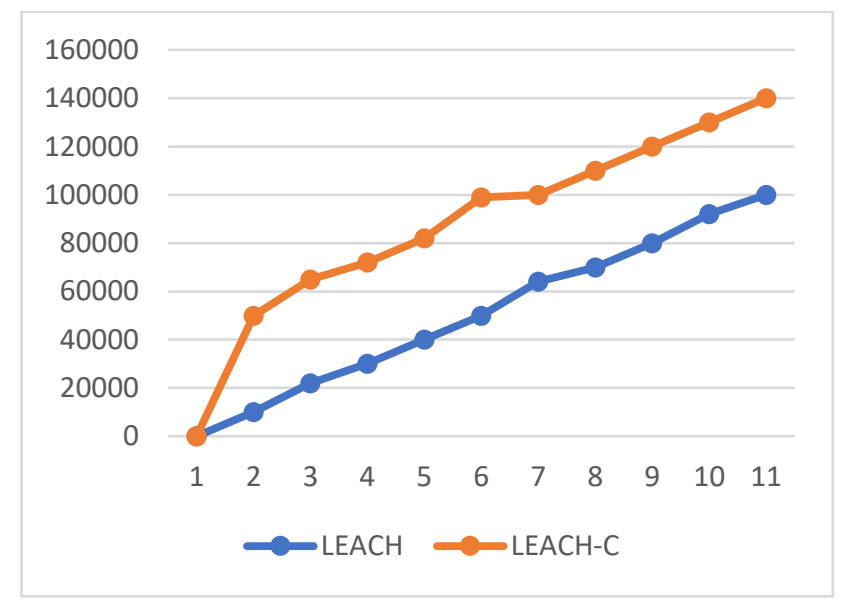

Figure 1 LEACH and LEACH-C in 1000kv SCADA station.
It is necessary to also figured out the performance in presence of NLOS scenario as shown in Figure 2. In an observational study the LEACH-C provide a huge amount of data from sensor nodes to base station as compared to LEACH protocol. This is because of having enough data items at SCADA system pinging them respace to BS. The cluster head will always remain same where data is not provided in a timed fashion. The drawback of this method is that the system as well as having a cluster head over the network the entire system will be affected if there is no node in a cluster head or a cluster head loss its energy. To avoid this problem a node must be with a enough power and energy from outer source.

\section{Conculusion}

The main challenge of this study was to analyze the performance of LEACH and LEACH-C protocol for $1000 \mathrm{kv}$ electric SCADA system in smart gridding environment. To achieve the full performance of WSN data utilization it is obvious to analyze the energy source of nodes and to check the performance of WSN nodes. In future work we are still investigating the performance of all routing protocols and present the result in the next phase of the study. Furthermore, we are also designing a complete routing protocol that will provide efficient results in presence of low powered sensor nodes.

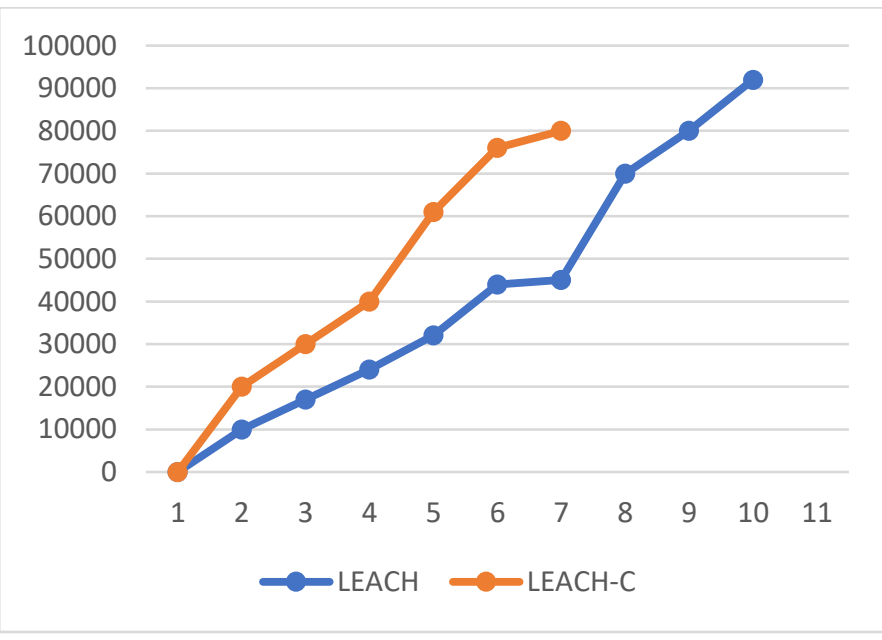

Figure 2 LEACH and LEACH-C in 1000kv SCADA station in presence of NLOS scenario.

\section{REFERENCES}

[1] Ahmad, Tanveer, Xue Jun Li, and Boon-Chong Seet. "3D localization based on parametric loop division and subdivision surfaces for wireless sensor networks." In 2016 25th Wireless and Optical Communication Conference (WOCC), pp. 1-6. IEEE, 2016.

[2] Ahmad, Tanveer, Xue Jun Li, and Boon-Chong Seet. "3D Localization Using Social Network Analysis for Wireless Sensor Networks." In 2018 IEEE 3rd International Conference on Communication and Information Systems (ICCIS), pp. 88-92. IEEE, 2018.

[3] M. Hammoudeh and R. Newman, "Adaptive routing in wireless sensor networks: QoSoptimisation for enhanced application performance," Information Fusion, Vol. 22, 
pp. $\quad$ 03-15, $\quad$ March 2015, https://doi.org/10.1016/j.inffus.2013.02.005.

[4] Ahmad, T., Li, X. J., \& Seet, B. C. (2017). Parametric loop division for 3D localization in wireless sensor networks. Sensors, 17(7), 1697.

[5] F. Farazandeh, R. Abrishambaf and S. Uysal, "A Hybrid Energy-Efficient routing protocol for Wireless Sensor Networks," in 11th IEEE International Conference on Industrial Informatics (INDIN), Bochum, Germany, 2013, DOI: 10.1109/INDIN.2013.6622851.

[6] P. Yuvaraj and KomanapalliVenkata Lakshmi Narayana, "EESCA: Energy efficient structured International Journal of Advanced Smart Sensor Network Systems (IJASSN), Vol 8, No.1/2/3, July 201814 clustering algorithm for wireless sensor networks," in International Conference on Computing, Analytics and Security Trends (CAST), Pune, India, 19-21 Dec. 2016, DOI: 10.1109/CAST.2016.7915024.

[7] S. C. Sharma and S. P. Singh, "A Survey on Cluster Based Routing Protocols in Wireless Sensor Networks," Procedia Computer Science, vol. 45, pp. 687-695, 2015, https://doi.org/10.1016/j.procs.2015.03.133.

[8] Z. A. Sadouq, M. El Mabrouk and M. Essaaidi, "Conserving Energy in WSN Through Clustering and Power Control," in Third IEEE International Colloquium in Information Science and Technology (CIST), Tetouan, Morocco, 20-22 Oct. 2014, DOI: 10.1109/CIST.2014.7016654.

[9] N. Ramluckun and V. Bassoo, "Energy-efficient chaincluster based intelligent routing technique for Wireless Sensor Networks," Applied Computing and Informatics, 07 March 2018, https://doi.org/10.1016/j.aci.2018.02.004.

[10]Ahmad, Tanveer, Xue Jun Li, and Boon-Chong Seet. "Fuzzy-Logic Based Localization for Mobile Sensor Networks." In 2019 2nd International Conference on Communication, Computing and Digital systems (CCODE), pp. 43-47. IEEE, 2019.

[11] M. Tripathi, M. S. Gaur, V. Laxmi and R. B. Battula, "Energy efficient LEACH-C protocol for Wireless Sensor Network," in CIIT 2013. Third International Conference on Computational Intelligence and Information Technology, Mumbai, India, 18-19 Oct. 2013, DOI: 10.1049/cp.2013.2620.
[12]L. Nassef, "On the Effects of Fading and Mobility in On Demand Routing Protocols," Egyptian Informatics Journal, Vol.11, pp. 67-74, 2010, http://dx.doi.org/10.1016/j.eij.2010.10.003.

[13] Heinzelman, Wendi Rabiner, AnanthaChandrakasan, and Hari Balakrishnan. "Energy-efficient communication protocol for wireless microsensor networks." In Proceedings of the 33rd annual Hawaii international conference on system sciences, pp. 10-pp. IEEE, 2000.

[14] Xiangning, Fan, and Song Yulin. "Improvement on LEACH protocol of wireless sensor network." In 2007 International Conference on Sensor Technologies and Applications (SENSORCOMM 2007), pp. 260-264. IEEE, 2007.

[15] Xinhua, $\mathrm{Wu}$, and Wang Sheng. "Performance comparison of LEACH and LEACH-C protocols by NS2." In 2010 Ninth International Symposium on Distributed Computing and Applications to Business, Engineering and Science, pp. 254-258. IEEE, 2010.

[16] Ahmad, Tanveer, Xue Jun Li, and Boon-Chong Seet. "Noise Reduction Scheme for Parametric Loop Division 3D Wireless Localization Algorithm Based on Extended Kalman Filtering." Journal of Sensor and Actuator Networks 8, no. 2 (2019): 24.

[17] V. Geetha, P. V. Kallapur and S. Tellajeera, "Clustering in Wireless Sensor Networks: Performance Comparison of LEACH \& LEACH-C Protocols Using NS2," Procedia Technology, Vol. 4, pp. 163170, 2012, https://doi.org/10.1016/j.protcy.2012.05.024.

[18] W. Xinhua and W. Sheng, "Performance comparison of LEACH and LEACH-C protocols by NS2," in Ninth International Symposium on Distributed Computing and Applications to Business Engineering and Science (DCABES), Hong Kong, China, 10-12 Aug. 2010.

[19] Ahmad, Tanveer. "An improved accelerated frame slotted aloha (afsa) algorithm for tag collision in rfid." arXiv preprint arXiv:1405.6217 (2014).

[20] Ahmad, T., Li, X. J., \& Seet, B. C. (2016, June). A selfcalibrated centroid localization algorithm for indoor ZigBee WSNs. In 2016 8th IEEE International Conference on Communication Software and Networks (ICCSN) (pp. 455-461). 